\title{
Effect of SOX2 on odontoblast differentiation of dental pulp stem cells
}

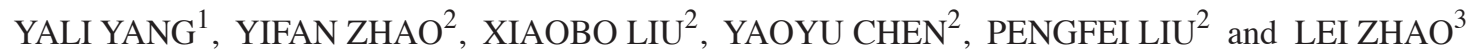 \\ ${ }^{1}$ Department of Anesthesiology, Henan Provincial People's Hospital, People's Hospital of Zhengzhou University, \\ Zhengzhou, Henan 450000; ${ }^{2}$ Department of Regenerative Medicine, School of Pharmaceutical Sciences, \\ Jilin University, Changchun, Jilin 130021; ${ }^{3}$ Department of Anesthesiology, Shenzhen People's Hospital, \\ Second Clinical Medical College of Jinan University, Shenzhen, Guangdong 518020, P.R. China
}

Received February 28, 2017; Accepted August 15, 2017

DOI: $10.3892 / \mathrm{mmr} .2017 .7812$

\begin{abstract}
The present study aimed to investigate the effect of SOX2 on odontoblast differentiation of dental pulp stem cells (DPSCs), and to examine the potential application of DPSCs on dental restoration. SRY-box 2 (SOX2) overexpression in human DPSCs (DPSCs-SOX2) was established by retroviral infection. The empty vector-infected DPSCs were regarded as a control group (DPSCs-vector group). Odontoblast differentiation culture medium was used to induce the differentiation of DPSCs. The degree of odontoblast differentiation of DPSCs was analyzed by flow cytometry. A genomic expression microarray and reverse transcription-quantitative polymerase chain reaction were used to analyze the molecular mechanism of SOX2 affecting odontoblast differentiation of DPSCs. After induction by odontoblast differentiation culture medium, the DPSC, DPSCs-vector and DPSCs-SOX2 groups all could carry out odontoblast differentiation. After induction for 3 weeks, all groups expressed dentin sialophosphoprotein (DSPP) and dentin matrix protein-1 (DMP-1) to a certain degree. However, after induction differentiation, the expression efficiency of DSPP and DMP-1 in the DPSCs-SOX2 group was highest compared with the normal DPSC and DPSCs-vector groups, which demonstrated the advantages of DPSC-SOX2 in odontoblast differentiation. Mechanism analysis indicated that in the DPSCs-SOX2 group, the activation of the Wnt signaling pathway and the upregulation of Wnt genes may be important mechanisms underlying SOX2 promoting odontoblast differentiation of DPSCs. These results preliminarily indicate that SOX2 has a certain promoting effect on odontoblast differentiation of DPSCs.
\end{abstract}

Correspondence to: Professor Lei Zhao, Department of Anesthesiology, Shenzhen People's Hospital, Second Clinical Medical College of Jinan University, 1017 North Dongmen Road, Shenzhen, Guangdong 518020, P.R. China

E-mail: leizhao1123@sohu.com

Key words: dental pulp stem cells, SRY-box 2, odontoblast differentiation, molecular mechanism, Wnt signaling pathway

\section{Introduction}

Dental pulp stem cells (DPSCs) are important mesenchymal stem cells in dental pulp tissue. Due to simple isolation and culture and certain amplification ability and multipotentiality DPSCs provide a new cell source for odontogenic tissue-engineered repair. The cell is usually obtained from naturally shedding teeth or extracted teeth in pathological situation, which provide a rich source, extremely promoting the application of DPSCs in the treatment of dental diseases (1-4). It has been proved that DPSCs have the ability of odontoblast differentiation. In the case of dentin or odontoblast damage, DPSCs can also differentiate into new functional odontoblasts to form dentin structure, accomplishing tooth restoration $(2,5)$. In recent years, how to optimize the function of DPSCs differentiating to odontoblasts and better achieve regeneration of dentin has been a hot issue in the field of oral medicine (4). The process of DPSCs differentiation involves many transcription factors associated with stem cell differentiation. Previous research has indicated that DPSCs serve the role of injury repair mainly by activating the Wnt signaling pathway (6). As an important transcription factor, SRY-box 2 (SOX2) has important significance in maintaining pluripotency of stem cells and somatic cell reprogramming (7-10). Simultaneously, it is also demonstrated in some research that SOX2 can induce neural differentiation of stem cells. In addition, SOX2, coupled with other transcription factors, can directly transform somatic cells without differentiation ability into nerve cells, which provides a novel technological solution to the cell therapy of nervous system disease $(11,12)$.

Our previous research indicated that the proliferation, migration and adhesion of DPSCs could were increased by SOX2 overexpression in DPSCs, which demonstrated that SOX2 has a certain effect on the biological characteristics of DPSCs (13). Though SOX2 can induce neural differentiation of stem cells at a certain degree, it is still not clear whether SOX2 has a certain effect on odontoblast differentiation of DPSCs. By referring to the previous research method (13), the present study was carried out to further explore the effect of SOX2 on odontoblast differentiation of DPSCs by regulating the Wnt signaling pathway. 


\section{Materials and methods}

Reagents and equipment. Dulbecco's modified Eagle's medium/F12, BME culture medium and fetal bovine serum (FBS) were purchased from Hyclone; Thermo Fisher Scientific, Inc. (Waltham, MA, USA). Sodium $\beta$-glycerophosphate, dexamethasone and vitamin $\mathrm{C}$ were bought from Sigma-Aldrich; Merck KGaA (Darmstadt, Germany). Transforming growth factor (TGF)- $\beta 1$ was purchased from R\&D Systems, Inc. (Minneapolis,MN,USA).TRIzol was purchased fromInvitrogen; Thermo Fisher Scientific, Inc. RNA reverse transcription kits and the quantitative polymerase chain reaction (qPCR) machine were bought from Takara Biotechnology Co., Ltd. (Dalian, China). All primer sequences were synthesized by Sangon Biotech Co., Ltd. (Shanghai, China; www.sangon.com/). Mouse anti-human dentin matrix protein-1 (DMP-1; cat. no. BS7995), anti-dentin sialophosphoprotein (DSPP; cat. no. BS71212) and anti-Wnt7b monoclonal antibodies (cat. no. BS71443) were purchased from Bioworld Technology, Inc. (St. Louis Park, MN, USA). A goat anti-mouse immunoglobulin (Ig) G-fluorescein isothiocyanate (FITC) polyclonal antibody (cat. no. BS20509) was bought from Invitrogen; Thermo Fisher Scientific, Inc. A human cell genomic expression microarray was purchased from Agilent Technologies, Inc. (Santa Clara, CA, USA).

An IX-70 inverted phase microscope was purchased from Olympus Corporation (Tokyo, Japan). PCR equipment was purchased from Eastwin Life Sciences, Inc. (Bejing, China; http://www.eastwin.com.cn/). CFX96 qPCR equipment was bought from Bio-Rad Laboratories, Inc. (Hercules, CA, USA) Company. A FACSCalibur flow cytometer was purchased from BD Biosciences (Franklin Lakes, NJ, USA).

Cell lines. Isolation and identification of primary DPSCs from human subjects was performed as described previously (13). A total of 37 patients ranging from 20-65 years old (16 males, 21 females) were screened, out of the patients treated in Henan Provincial People's Hospital (Zhengzhou, China) in December 2015 and the pulp tissue was collected. DPSCs were isolated and incubated under sterile conditions in research center of the Henan Provincial People's Hospital. The empty vector control-infected (DPSCs-vector) and the SOX2-overexpressing (DPSCs-SOX2) cell lines were generated as described previously (13), and the stable cell lines were used in the present study. The present study was approved by the ethics committee of Henan Provincial People's Hospital (Zhengzhou, China), and written informed consent was obtained from all patients.

Odontoblast differentiation of DPSCs. The method of odontoblast differentiation of DPSCs performed as described previously (14). DPSCs in the logarithmic growth phase were collected, and seeded into 24-well plates at a density of $2 \times 10^{5}$ cells/well. The cells were cultivated with odontoblast differentiation medium (10\% FBS, $10^{-3} \mathrm{~mm}$ dexamethasone, $10 \mathrm{~mm}$ sodium $\beta$-glycerophosphate, $50 \mathrm{mg} / \mathrm{l}$ vitamin $\mathrm{C}$ and $5 \mathrm{ng} / \mathrm{ml}$ TGF- $\beta 1$ were added into BME basal medium), and differentiated for 3 weeks.

Flow cytometry. After the induction and differentiation, flow cytometry was used to test the expression levels of DSPP and DMP-1 in the DPSC, DPSC-vector and DPSC-SOX2 groups to analyze the effect of odontoblast differentiation of DPSCs. Then
DPSCs in each experimental group were fixed and penetrated with $4 \%$ paraformaldehyde at room temperature with 20 mins, and mouse anti-human DSPP and DMP-1 monoclonal antibodies (1:50) in FBS (Hyclone; GE Healthcare Life Sciences, Logan, UT, USA) were incubated with cells for $30 \mathrm{~min}$ at room temperature. After cleaning twice with PBS, a goat anti-mouse IgG-FITC polyclonal antibody (1:400) was incubated with the cells for $30 \mathrm{~min}$ at room temperature. Following cleaning twice with PBS again, the cells were detected using a FACSCalibur flow cytometer (FACS101; BD Biosciences).

For analysis of Wnt7b expression, a mouse anti-human Wnt7b monoclonal antibody was diluted to $1: 50$, and a goat anti-mouse IgG-FITC polyclonal antibody was diluted to 1:400. The assay was performed at room temperature for $1 \mathrm{~h}$. After cleaning with PBS, these antibodies were detected using a FACSCalibur flow cytometer.

Microarray analysis. A microarray analysis comparing the gene expression profiles of the DPSCs-vector and the DPSCs-SOX2 cell lines was performed as previously described (13), and the results are publicly available in the Gene Expression Omnibus database (accession no. GSE73548).

Reverse transcription (RT)-qPCR. TRIzol was used to extract RNA from cells. cDNA was generated using an RNA reverse transcription kit. RT-qPCR was used to confirm the expression of genes in the Wnt signaling pathway that were identified as significantly altered by the previous microarray analysis but were not described or confirmed previously (15). Primer sequences for RT-qPCR were: Wnt1 forward, 5'-CGATGGTGG GGTATTGTGAAC-3' and reverse, 5'-CCGGATTTTGGCGTA TCAGAC-3'; Wnt7b forward, 5'-GAAGCAGGGCTACTACAA CCA-3' and reverse, 5'-CGGCCTCATTGTTATGCAGGT-3'; Wnt8a forward, 5'-GAACTGCCCTGAAAATGCTCT-3' and reverse, 5'-TCGAAGTCACCCATGCTACAG-3'; Wnt11 forward, 5'-GACCTCAAGACCCGATACCTG-3' and reverse; 5'-TAGACGAGTTCCGAGTCCTTC-3'; and $\beta$-actin forward, 5'-CCCAGAGCAAGAGAGG-3' and reverse, 5'-GTCCAGACG CAGGATG-3'. With the fluorophore SYBR Advantage qPCR Premix (cat. nos. 638321 and 639676; Takara Bio, Inc., Otsu, Japan), used so any alterations were clearly observable. The $2^{-\Delta \Delta C q}$ method was used for quantification (15). As the mRNA expression of Wnt7b was revealed to be the most significantly upregulated, the protein expression level of Wnt7b was subsequently assessed by flow cytometry. The thermocycling conditions were: Predenaturing at $95^{\circ} \mathrm{C}, 5 \mathrm{~min}$; denaturing at $94^{\circ} \mathrm{C}, 30 \mathrm{sec}$, annealing at $64^{\circ} \mathrm{C}, 30 \mathrm{sec}$, extension $\left(72^{\circ} \mathrm{C}, 30 \mathrm{sec}\right)$ with 32 cycles and terminal extension $\left(72^{\circ} \mathrm{C}, 5 \mathrm{~min}\right)$.

Statistical analysis. SPSS 17.0 (SPSS, Inc., Chicago, IL, USA) was used to analyze clinical data. Data are presented as mean \pm standard error. A student's t-test was used for comparison between groups, and two-way analysis of variance was used for multiple comparisons. $\mathrm{P}<0.05$ was considered to indicate a statistically significant difference.

\section{Results}

Odontoblast differentiation of DPSCs. In our previous study, isolated DPSCs were demonstrated to exhibit a mesenchymal 


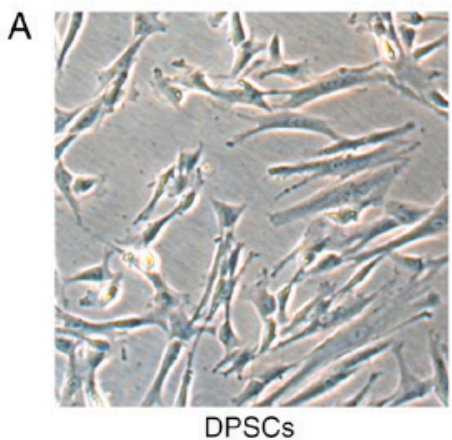

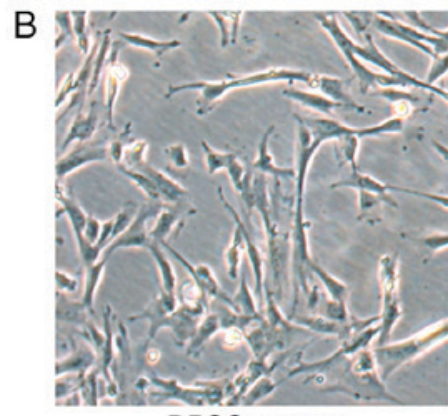

DPSCs-vector

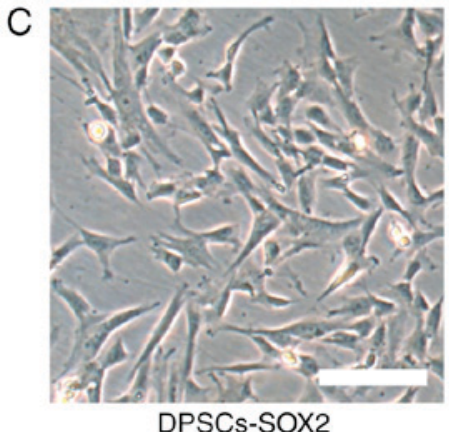

DPSCs-SOX2

Figure 1. Morphological characteristics of DPSCs in each group. Representative photomicrographs of DPSCs in the (A) DPSC, (B) DPSC-vector and (C) DPSC-SOX2 groups (scale bar, $200 \mu \mathrm{m}$ ). SOX2, SRY-box 2; DPSCs, dental pulp stem cells.

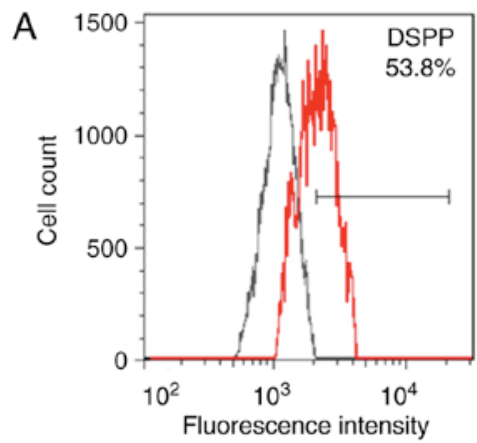

DPSCs

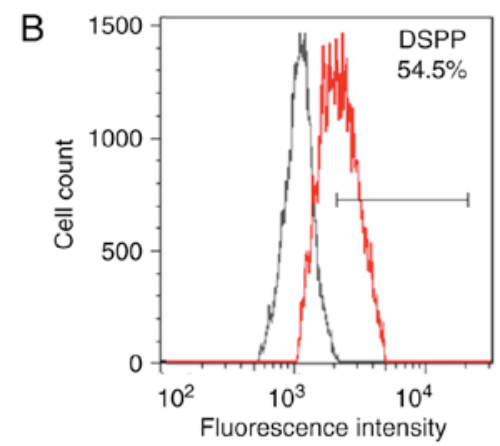

DPSCs-vector

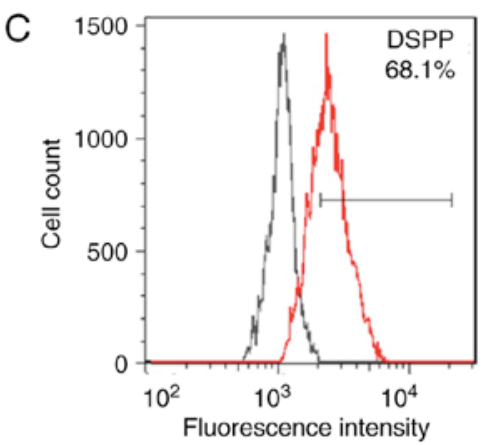

DPSCs-SOX2

Figure 2. Expression of DSPP after DPSC differentiation in each group. Flow cytometry analysis of DSPP expression in the (A) DPSC, (B) DPSC-vector and (C) DPSC-SOX2 groups. SOX2, SRY-box 2; DPSCs, dental pulp stem cells; DSPP, dentin sialophosphoprotein.
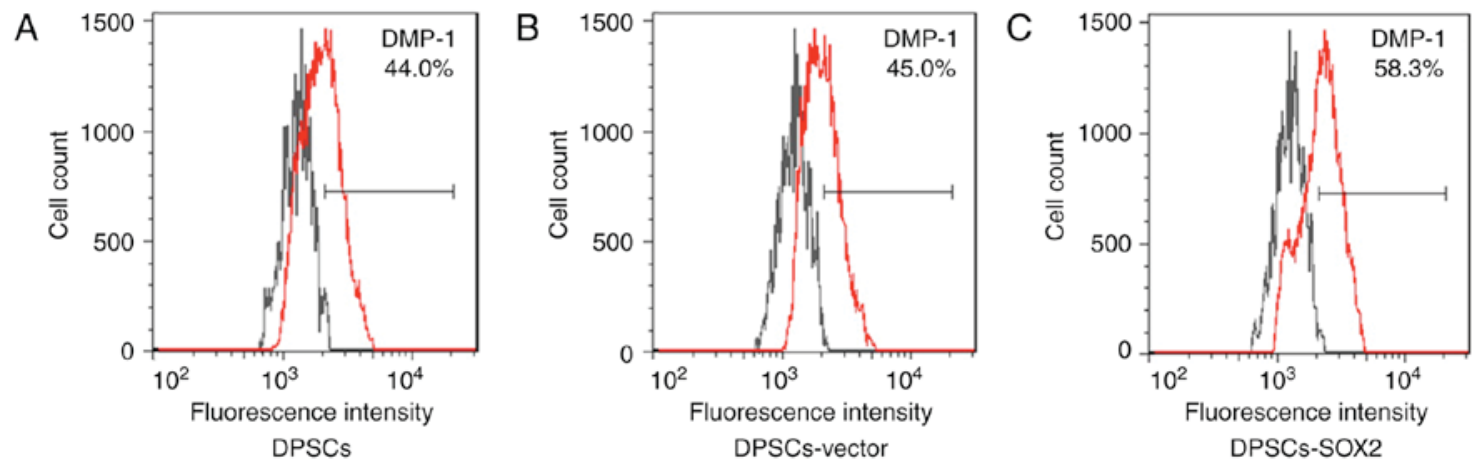

Figure 3. Expression of DMP-1 after DPSC differentiation in each group. Flow cytometry analysis of DMP-1 expression in the (A) DPSC, (B) DPSC-vector and (C) DPSC-SOX2 groups. SOX2, SRY-box 2; DPSCs, dental pulp stem cells; DMP-1, dentin matrix protein-1.

phenotype, to possess osteogenic and adipogenic differentiation potential, and to have increased proliferation, migration and adherence function following SOX2 overexpression (13). In the present study, the odontoblast differentiation ability among the normal DPSCs, DPSCs-vector and DPSCs-SOX2 groups was examined. The results revealed that there was no significant difference among normal DPSCs, DPSCs-vector and DPSCs-SOX2 groups in morphology, and that the cells in all groups grew by adherence in long spindle shapes (Fig. 1). After the induction of odontoblast differentiation, the cells in three groups could express the odontoblast special markers DSPP and DMP-1, but there was some difference in expression degree. After DPSCs-SOX2 was induced to

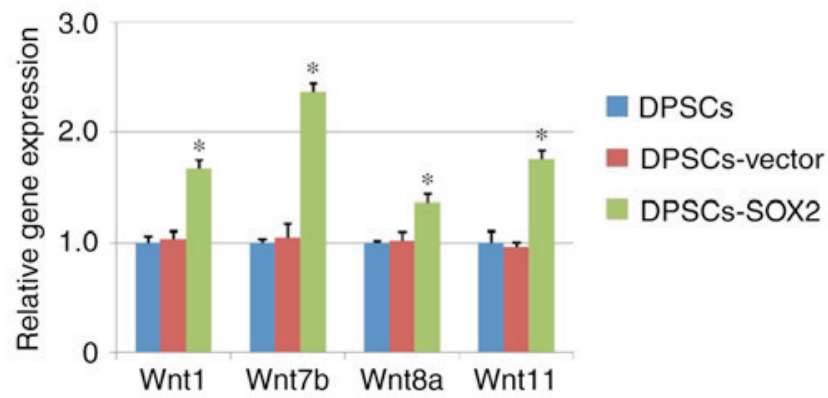

Figure 4. Detection of genes expression of the Wnt family. mRNA expression levels of Wnt family members in DPSCs. The data was presented as mean \pm standard error. "P<0.05 vs. DPSCs and DPSC-vector. SOX2, SRY-box 2; DPSCs, dental pulp stem cells. 

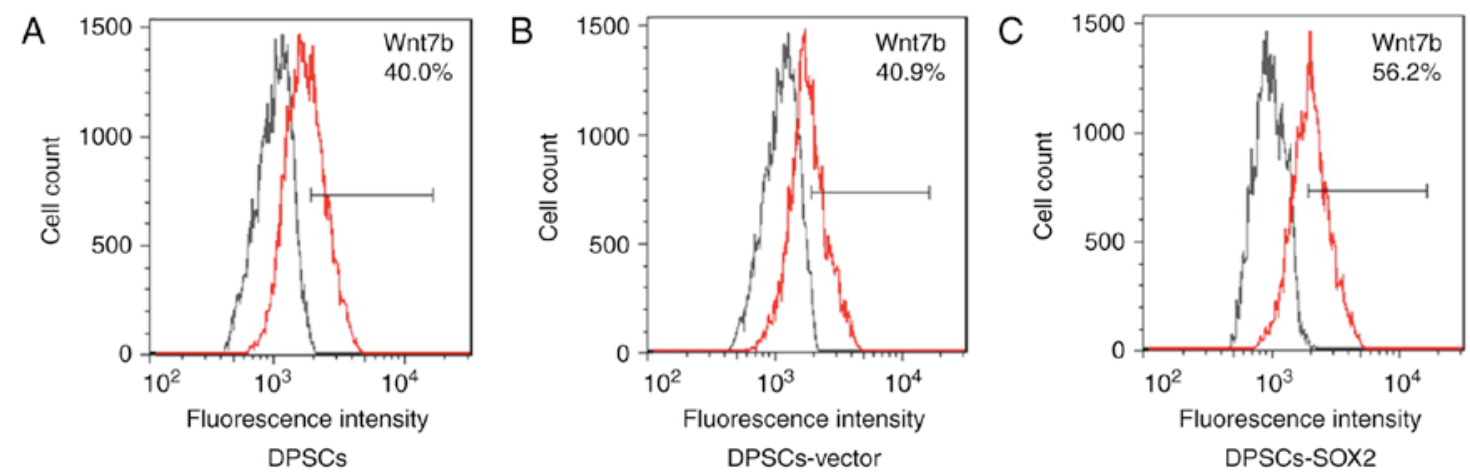

Figure 5. Flow cytometry analysis of Wnt7b expression. Wnt7b expression in the (A) DPSC, (B) DPSC-vector and (C) DPSC-SOX2 groups. SOX2, SRY-box 2; DPSCs, dental pulp stem cells.

odontoblast differentiation, the expression efficiency of DSPP in DPSCs-SOX2 group was 68.1\%, which was higher compared with the normal DPSC group (53.8\%) and in the DPSCs-vector group (54.5\%; $\mathrm{P}<0.05$; Fig. 2). The expression efficiency of DMP-1 in the DPSCs-SOX2 group was $58.3 \%$, which was higher than the DPSC group (44.0\%) and the DPSC-vector group (45.0\%; Fig. 3).

Analysis on mechanism of SOX2 affecting osteogenic differentiation of DPSCs. The change of signaling pathways in DPSCs after SOX2 overexpression was preliminarily evaluated by genomic expression microarray, and the effect of SOX2 on odontoblast differentiation of DPSCs was analyzed. The results from a previously published microarray analysis comparing the expression profiles of the DPSCs-vector and DPSCs-SOX2 cells indicated that several genes involved in the Wnt pathway were significantly upregulated following SOX2 overexpression (16). The Wnt signaling pathway serves an important role in regulating function in tooth development, and also has a significant effect on dentinal formation (17). Therefore, the Wnt signalling pathway may have a role in the SOX2-mediated induction of odontoblast differentiation.

According to analysis results of genome expression microarray, promoter genes (Wnt1, Wnt7b, Wnt8a and Wnt11) in the Wnt signaling pathway were evaluated by RT-qPCR and flow cytometry. The results of RT-qPCR demonstrated that Wnt1, Wnt7b, Wnt8a and Wnt11 in the DPSCs-SOX2 group all were upregulated compared with the DPSC and DPSCs-vector groups. Among these promoter genes, the upregulation of Wnt7b was highest (Fig. 4). Therefore, the expression level of Wnt7b at the protein level in each experimental group was further analyzed by flow cytometry. The results demonstrated that the expression Wnt7b protein in each experimental group was consistent with the result of RT-qPCR. The expression efficacy of Wnt7b in the DPSCs-SOX2 group (56.2\%) was highest compared with the DPSC (40.0\%) and DPSC-vector (40.9\%) groups (Fig. 5)

\section{Discussion}

Previous studies have demonstrated that DPSCs has an ability of odontoblast differentiation. DPSCs serve an important role in dentin regeneration and injury repair, especially in the case of tooth injury (1-4). However, as a pluripotent regulatory gene and a neurogenic differentiation factor, it is still unclear about the effect of SOX2 on odontoblast differentiation of DPSCs. This study was carried out to investigate this issue. On the basis of differentiation experiments and mechanism analysis, the experimental results in this research primarily demonstrated that SOX2 overexpression in DPSCs could upregulate the expression of DSPP and DMP-1, and promote odontoblast differentiation of DPSCs. Simultaneously, it was demonstrated by microarray analysis that the Wnt signaling pathway was more active in the DPSCs-SOX2 group compared with the DPSC group; many key genes were upregulated, which was also revealed by RT-qPCR and flow cytometry. The activation of the Wnt signaling pathway might be an important mechanism of SOX2 promoting the odontoblast differentiation of DPSCs.

The Wnt signaling pathway has an important effect on tooth development, and is an important signal in the process of dentin formation $(18,19)$. By using techniques like microarray, the present results indicated that Wnt1, Wnt7b, Wnt8a and Wnt11 all were upregulated following SOX2 overexpression. These factors are activators in the Wnt signaling pathway, which could effectively activate downstream genes in Wnt signaling $(20,21)$. Previous research has demonstrated that overexpression of Wnt signaling molecules in DPSCs is beneficial for their odontoblast differentiation (22). Therefore, the Wnt signaling pathway may be a vital approach for SOX2 affecting odontoblast differentiation of DPSCs. However, it is still unclear whether the upregulation of Wnt signaling molecules is influenced by SOX2 directly or indirectly. It is necessary to further analyze the issue. If the issue is solved, it will be more conducive to the development of novel technical approaches in order to effectively regulate and control odontoblast differentiation of DPSCs.

In conclusion, the results of present study demonstrated that SOX2 overexpression in DPSCs promoted the regulation of odontoblast differentiation of DPSCs and provided a theoretical basis for the application of DPSCs in dental pulp injury repair. However, this study mainly assessed the underlying mechanisms in vitro; further in vivo studies are required to investigate the odontoblast differentiation and injury repair ability of DPSCs for directly transplanting DPSCs-SOX2 to damaged teeth.

\section{Acknowledgements}

The authors would like to thank all those who have helped during the writing of this manuscript, and gratefully acknowledge 
the help of Professor Zhao Lei in his patience, encouragement, and professional instructions during manuscript writing.

\section{References}

1. Chang CC, Chang KC, Tsai SJ, Chang HH and Lin CP: Neurogenic differentiation of dental pulp stem cells to neuron-like cells in dopaminergic and motor neuronal inductive media. J Formos Med Assoc 113: 956-965, 2014.

2. Gronthos S, Mankani M, Brahim J, Robey PG and Shi S: Postnatal human dental pulp stem cells (DPSCs) in vitro and in vivo. Proc Natl Acad Sci USA 97: 13625-13630, 2000.

3. Paino F, La Noce M, Tirino V, Naddeo P, Desiderio V, Pirozzi G, De Rosa A, Laino L, Altucci L and Papaccio G: Histone deacetylase inhibition with valproic acid downregulates osteocalcin gene expression in human dental pulp stem cells and osteoblasts: Evidence for HDAC2 involvement. Stem Cells 32: 279-289, 2014.

4. Tatullo M, Marrelli M, Shakesheff KM and White LJ: Dental pulp stem cells: Function, isolation and applications in regenerative medicine. J Tissue Eng Regen Med 9: 1205-1216, 2015.

5. Zheng Y, Wang XY, Wang YM, Liu XY, Zhang CM, Hou BX and Wang SL: Dentin regeneration using deciduous pulp stem/progenitor cells. J Dent Res 91: 676-682, 2012.

6. Bakopoulou A, Leyhausen G, Volk J, Papachristou E, Koidis P and Geurtsen W: Wnt/ $\beta$-catenin signaling regulates Dental Pulp Stem Cells' responses to pulp injury by resinous monomers. Dent Mater 31: 542-555, 2015.

7. Cai J, Li W, Su H, Qin D, Yang J, Zhu F, Xu J, He W, Guo X, Labuda K, et al: Generation of human induced pluripotent stem cells from umbilical cord matrix and amniotic membrane mesenchymal cells. J Biol Chem 285: 11227-11234, 2010.

8. Esteban MA, Wang T, Qin B, Yang J, Qin D, Cai J, Li W, Weng Z, Chen J, Ni S, et al: Vitamin C enhances the generation of mouse and human induced pluripotent stem cells. Cell Stem Cell 6: 71-79, 2010.

9. Takahashi K, Tanabe K, Ohnuki M, Narita M, Ichisaka T, Tomoda $\mathrm{K}$ and Yamanaka S: Induction of pluripotent stem cells from adult human fibroblasts by defined factors. Cell 131 $861-872,2007$

10. Takahashi $\mathrm{K}$ and Yamanaka S: Induction of pluripotent stem cells from mouse embryonic and adult fibroblast cultures by defined factors. Cell 126: 663-676, 2006.
11. Feng R and Wen J: Overview of the roles of Sox 2 in stem cell and development. Biol Chem 396: 883-891, 2015.

12. Thier M, Wörsdörfer P, Lakes YB, Gorris R, Herms S, Opitz T, Seiferling D, Quandel T, Hoffmann P, Nöthen MM, et al: Direct conversion of fibroblasts into stably expandable neural stem cells. Cell Stem Cell 10: 473-479, 2012.

13. Liu P, Cai J, Dong D, Chen Y, Liu X, Wang Y and Zhou Y: Effects of SOX2 on proliferation, migration and adhesion of human dental pulp stem cells. PLoS One 10: e0141346, 2015.

14. Yang KC, Kitamura Y, Wu CC, Chang HH, Ling TY and Kuo TF: Tooth germ-like construct transplantation for whole-tooth regeneration: An in vivo study in the miniature pig. Artif Organs 40: E39-E50, 2016.

15. Livak KJ and Schmittgen TD: Analysis of relative gene expression data using real-time quantitative PCR and the 2(-Delta Delta C(T)) method. Methods 25: 402-408, 2001.

16. Lin X, Dong R, Diao S, Yu G, Wang L, Li J and Fan Z: SFRP2 enhanced the adipogenic and neuronal differentiation potentials of stem cells from apical papilla. Cell Biol Int 41: 534-543, 2017.

17. Liu F and Millar SE: Wnt/beta-catenin signaling in oral tissue development and disease. J Dent Res 89: 318-330, 2010.

18. Liu B, Chen S, Cheng D, Jing W and Helms JA: Primary cilia integrate hedgehog and Wnt signaling during tooth development. J Dent Res 93: 475-482, 2014

19. Yuan G, Yang G, Zheng Y, Zhu X, Chen Z, Zhang Z and Chen Y: The non-canonical BMP and Wnt/ $\beta$-catenin signaling pathways orchestrate early tooth development. Development 142: 128-139, 2015.

20. Maye P, Zheng J, Li L and Wu D: Multiple mechanisms for Wnt11-mediated repression of the canonical Wnt signaling pathway. J Biol Chem 279: 24659-24665, 2004.

21. Katoh M: Regulation of WNT signaling molecules by retinoic acid during neuronal differentiation in NT2 cells: Threshold model of WNT action (Review). Int J Mol Med 10: 683-687, 2002.

22. Koizumi Y, Kawashima N, Yamamoto M, Takimoto K, Zhou M, Suzuki N, Saito M, Harada H and Suda H: Wnt11 expression in rat dental pulp and promotional effects of Wnt signaling on odontoblast differentiation. Congenit Anom (Kyoto) 53: 101-108, 2013. 